\title{
Dynamic changes in antibody levels as an early warning of Salmonella Dublin in bovine dairy herds
}

\author{
A. Stockmarr, ${ }^{*}{ }^{1}{ }^{R}$. Bødker, ${ }^{*}$ and L. R. Nielsen $\ddagger$ \\ *National Veterinary Institute, Technical University of Denmark, Bülowsvej 27, DK-1870 Frederiksberg C, Denmark \\ †Department of Applied Mathematics and Computer Science, Technical University of Denmark, Matematiktorvet, Building 303B, DK-2800 Lyngby, \\ Denmark \\ ‡Department of Large Animal Sciences, Faculty of Health and Medical Sciences, University of Copenhagen, Grønnegårdsvej 8 , \\ DK-1870 Frederiksberg C, Denmark
}

\section{ABSTRACT}

Salmonella Dublin is a bacterium that causes disease and production losses in cattle herds. In Denmark, a surveillance and control program was initiated in 2002 to monitor and reduce the prevalence of Salmonella Dublin. In dairy herds, the surveillance includes herd classification based on bulk tank milk measurements of antibodies directed against Salmonella Dublin at 3-mo intervals. In this study, an "alarm herd" concept, based on the dynamic progression of these repeated measurements, was formulated such that it contains predictive power for Salmonella Dublin herd classification change from "likely free of infection" to "likely infected" in the following quarter of the year, thus warning the farmer 3 mo earlier than the present system. The alarm herd concept was defined through aberrations from a stable development over time of antibody levels. For suitable parameter choices, alarm herd status was a positive predictor for Salmonella Dublin status change in dairy herds, in that alarm herds had a higher risk of changing status in the following quarter compared with nonalarm herds. This was despite the fact that both alarm and nonalarm herds had antibody levels that did not indicate the herds being "likely infected" according to the existing classification system in the present quarter. The alarm herd concept can be used as a new early warning element in the existing surveillance program. Additionally, to improve accuracy of herd classification, the alarm herd concept could be incorporated into a model including other known risk factors for change in herd classification. Furthermore, the model could be extended to other diseases monitored in similar ways.

Key words: Salmonella, classification, statistical model, early warning

Received December 13, 2012.

Accepted September 9, 2013.

${ }^{1}$ Corresponding author: anst@dtu.dk

\section{INTRODUCTION}

Salmonella enterica ssp. enterica serovar Dublin (Salmonella Dublin) is a bacterium that leads to compromised animal health and production losses in cattle herds worldwide (McDonough et al., 1999; CarriqueMas et al., 2010). Therefore, several countries have initiated research and extended programs for screening and control of this infection in their cattle populations (Veling et al., 2002; Bergevoet et al., 2009; Lewerin et al., 2011).

In Denmark, a national surveillance program was initiated in 2002 for Salmonella Dublin. All dairy herds are classified based on the level of antibodies against Salmonella Dublin measured in bulk tank milk samples collected automatically through a milk quality assurance program (Anonymous, 2004). Antibody levels are quantified as background-corrected optical density calibrated (ODC\%) values, as described by Nielsen and Ersbøll (2005). A herd status of "likely free of Salmonella Dublin" (status 1) is given in the current surveillance program if (1) the average of the last 4 ODC\% sample values, where samples were collected with at least $3 \mathrm{wk}$ between them, is below 25; and (2) the ODC\% value of the newest sample has not increased more than 20 percentage points from the average of the preceding 3 samples.

If status 1 is not given, the herd is given status 2: "likely infected with Salmonella Dublin". No microbiological follow-up is performed upon a status 2 assignment. Other classifications are possible, but they are rare for dairy herds and outside the scope of the presented work. More detailed information about the surveillance program and an evaluation of the validity and predictive values of the current classification system are available in Warnick et al. (2006).

With quarterly measurements as a standard, on average 3 mo elapses between measurements, leading to an inherent delay in status changes in newly infected herds (Jordan et al., 2008). Previous studies have analyzed risk factors for changing status from 1 to 2 (Nielsen et 
al., 2007; Ersbøll and Nielsen, 2008). However, these models were based on present or recent characteristics for the herds and did not take into account previous antibody measurements from the herds. The need exists, therefore, for characteristics with predictive power, so that characteristics at the previous measurement time points will indicate a risk of development toward a status 2 classification at the next measurement time. An obvious characteristic is the average level of antibody measurements for the 3 most recent samples, which enters the definition of the requirements for status 1 . However, this characteristic does not necessarily yield information about the dynamic progression in the bulk tank milk samples. For a status 1 herd with stable development of antibody measurements around a certain level (this being low or high but not so high that it conflicts with the status 1 classification), an upward deviation may indicate a disturbance, which could be caused by a yet-undetected Salmonella Dublin infection in an early stage of an outbreak.

Herds at risk of changing status and showing such an upward deviation in antibody measurements could be identified earlier than in the existing surveillance system, and farmers would be able to take appropriate action toward a possible spread of infection within and away from their herds. This would benefit the aim of reducing Salmonella Dublin prevalence nationally.

The purpose of this study was to define "alarm herds" as a register-based early warning concept for herds at a higher risk of changing Salmonella Dublin status from status 1 to status 2 in the following quarter of the year, through upward dynamic deviations in antibody measurements, and to investigate the effect of this concept on early detection.

\section{MATERIALS AND METHODS}

\section{Data Collection}

Bulk tank milk antibody measurements collected as part of the national surveillance program for Salmonella Dublin were obtained from the Danish Cattle Database for all Danish dairy herds from 2001 to 2007. The bulk tank milk samples were collected automatically approximately every 3 mo through a mandatory milk quality control scheme organized by the Danish Cattle Federation (Aarhus, Denmark). The samples were tested by using an ELISA for IgG directed against Salmonella Dublin using an antigen produced by the National Veterinary Institute (Technical University of Denmark, Frederiksberg). The test is an indirect in-house ELISA used at Eurofins/Steins Laboratory (Holstebro, Denmark). The test procedures have been described in detail by Nielsen and Ersbøll (2005). The level of antibodies in the samples were estimated on a semiquantitative scale as background-corrected optical density proportions of positive control samples on the same ELISA plate (ODC\%). All values $<0$ ODC\% were censored to the value of zero, resulting in a range of 0 to 213 ODC\%. These values were used to determine the average ODC\% value and the herd Salmonella Dublin status for each quarter of the year. For Salmonella Dublin status, we based our analyses on the average quarterly ODC\% values, and followed the official classification system. However, we applied a slightly different classification, where a lack of samples in a quarter was interpreted as missing data. We used the arithmetic mean for the average ODC\% values when more than one sample was reported in a quarter.

The average ODC\% values were treated as observations in the following analyses. In total, 179,410 observations were available from 9,387 dairy herds. On average, the herds included in the study had 19 observations in the study period from 2001 to 2007. This is lower than the total number of year-quarters covered, because many herds went out of business during that period.

\section{Stability, Jumps, and Alarm Herds: Definitions}

"Stable development" was defined as ODC\% values not varying more than a normal distribution with the same mean and variance would. Normality of ODC\% values was not assumed, but in a period of stable development, we required the ODC\% values to be contained in a standard $95 \%$ CI around their mean, where the confidence interval was based on a normal distribution approximation with mean and variance equal to mean and variance of the considered data. For this purpose, we constructed a "stability area," indicating a stable development of ODC\% measurements, as a symmetric area around the mean of preceding observations as follows.

Sample data from each herd were considered as a time series, and we initially investigated whether the first 4 observations, corresponding to the quarters in 2001, were available. If so, we checked whether these data resulted in a status 1 classification as explained above; if they did, the empirical mean $\hat{\mu}$ of these 4 was calculated. A preliminary confidence region for the data was then constructed using the following standard result.

For independent identically distributed normal random variables $Y_{1}, \ldots, Y_{n}$, it holds that the $p$-percentile $q_{p, \max }$ for $\max \left(Y_{1}, \ldots, Y_{n}\right)$ is found as $q_{p, \max }=q_{p^{1 / n}}$, where $q_{p^{1 / n}}$ is the $p^{1 / n}$-th percentile for the standard normal distribution, and $\sigma^{2}$ is the variance of $Y_{1}$. We 
assumed that the 4 observations varied around a common mean estimated by $\hat{\mu}$, and their common variance was estimated by the empirical variance $\hat{\sigma}^{2}$ (a minimum variance of 1 was entered if the empirical variance was $<1$ ). Using the fact that the deviation from the empirical mean $\hat{\mu}$ has a variance of $3 \sigma^{2} / 4$, we obtained the preliminary confidence region limits $\hat{\mu} \pm \sqrt{4 / 3} \hat{\sigma} q_{t(3), 0.975^{1 / 4}}$, where $q_{t(3), r}$ is the $r$ th percentile in the $t$ distribution with 3 degrees of freedom. We note that $\sqrt{4 / 3} q_{t(3), 0.975^{1 / 4}} \approx 6.20$. If all 4 observations were within this preliminary confidence region around $\hat{\mu}$, the mean and variance were estimated from all 3 sets of the 4 data points, and we investigated whether all 4 data points were within the corresponding preliminary confidence regions, to ensure that a single outlying point did not drive up the 4 -set variance. If all 4 data points were within all 4 confidence regions, the most recent time point was termed a "stable" time point for the herd in question. The stability area for a new observation was then calculated as the interval with endpoints $\hat{\mu} \pm \sigma^{2} q_{t(4), 0.975}$.

All time points that were not stable were termed "unstable." Because variances were not calculated when fewer than 4 observations were available, a burn-in phase with formally unstable time points necessarily had to be present before stable time points occurred.

Moving on to the next time point (first quarter of 2002), we investigated whether this fifth data point (if available) resulted in a status 1 classification. If so, we investigated, for herds that were unstable at the previous time point (fourth quarter of 2001), whether the new data point together with earlier available time points made the current time point a stable point. For herds that were stable at the previous time point, we investigated whether the present data point (at time 5) was within the stability area calculated at the previous time point. If it was, the time point 5 was termed stable. The empirical mean and variance, and thus the stability area, were updated with this observation, and the next observation was investigated in the same way. If the observation at time 5 was outside the stability area, the time point was termed unstable, and we would say that a "jump" had occurred. Following a jump, the procedure above was repeated, with "earlier available time points" being reset to be all points following the most recent stable time point, to the end of the time series.

We were interested in jumps that indicated an increased risk of changing Salmonella Dublin status to status 2 ; thus, not all jumps were interesting. To increase the risk, jumps needed to be to a level above the stability area, because jumps where the time series decreased to a lower level were assumed not to indicate an increased risk for changing from Salmonella Dublin status 1 to status 2. Also, jumps to a very low level would likely not increase the risk of changing Salmonella Dublin status significantly, even though the level would be higher than what the stability area covered. To take these possibilities into account, we parameterized the level from which the jump would be assumed to increase the risk of a status change, by introducing the alarm threshold $(c)$. If the time series of bulk tank milk measurements at a time point $t$ jumped outside the stability area to a level higher than the alarm threshold $c$, we defined the jump to be an "alarm jump" and denoted the herd as an alarm herd at time $t$. Note that we considered the level that the ODC\% jumped to, rather than the size (i.e., the difference to the previous level) of the jump. This procedure is illustrated in Figure 1, where 3 stability periods, of length 6,1 , and 6 quarters, respectively, are established. A single upward jump was present at the 10th quarter. This jump was an alarm jump if the alarm threshold was at 13 or below. The ODC $\%$ value at quarter 15 triggered a status 2 classification. It should be noted from the described procedure that it is implicit in the definition of a jump that it does not trigger a status 2 classification. Hence, the value at quarter 15 was not considered a jump.

The total number of alarm jumps detected in the study period is depicted in Figure 2 as a function of the alarm threshold $c$. For $c=0$ (i.e., no threshold, all jumps are alarm jumps), 6,537 alarm jumps were detected. For $c=20$, the number of alarm jumps detected was 455 . The largest detected alarm jump was to a value of 39 .

For values of $c$ between 0 and 25, we investigated whether alarm jumps with an alarm threshold of $c$ constituted a statistically significant predictor for Salmonella Dublin classification change from status 1 to status 2, disregarding any other information. A standard logistic regression model, with herd classification status at time $t$ as response and alarm herd status at time $t-1$ as explanatory variable, was fitted to the empirical Salmonella Dublin status for each herd and each year-quarter in the study period, disregarding the first 5 year-quarters where no alarm herd status at the previous quarter could be established. Tests of effects of alarm herd status were carried out as likelihood ratio tests, evaluated with $\chi^{2}$ distributions. The effect of small jumps; that is, upward jumps that were not considered alarm jumps, was investigated in a similar way.

\section{RESULTS}

The proportion of herds classified as status 2 herds in the year-quarter after they became alarm herds 


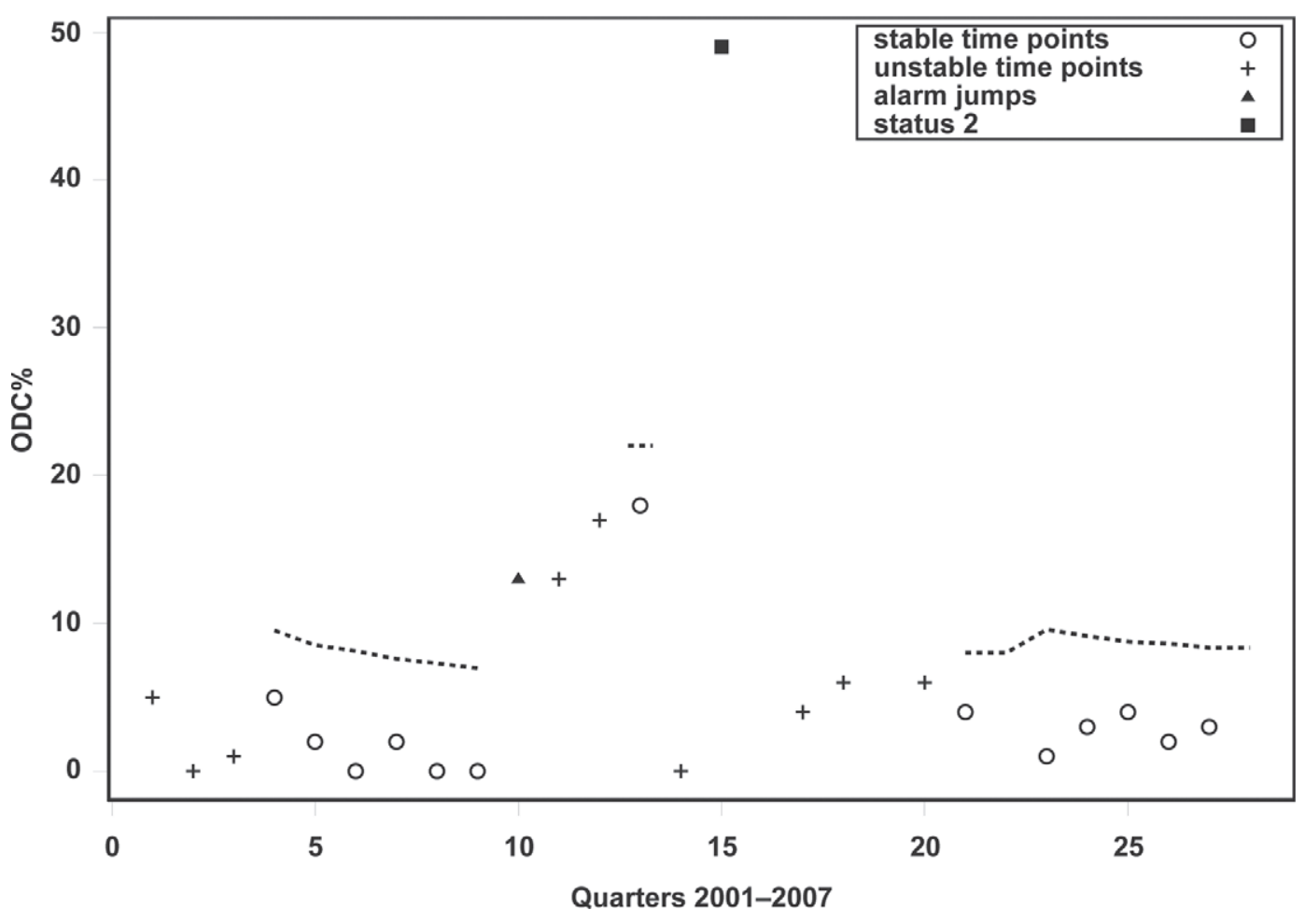

Figure 1. Time series for a dairy herd showing the development in stability evaluation of Salmonella Dublin bulk tank measurements. Data from the Danish national surveillance program between 2001 and 2007. The upper bound for the stability area is shown as a dashed line above the stable time points. The alarm jump at time 10 (second quarter of 2003) is relative to an alarm threshold $c$ of 13 or below. ODC $=$ optical density calibration.

increased with the alarm threshold $c$ (Figure 3). The proportion was 0.023 for $c=0$, increasing to 0.084 for $c$ $=20$, and to 0.121 for $c=25$. This should be compared with the overall proportion of status 1 herds being classified as a status 2 herd next quarter during the study period, which was estimated empirically to 0.016 . The increase in proportion of herds with subsequent change to status 2 with increasing alarm threshold was seen to be markedly higher for $c \geq 5$, and even higher for $c>$ 20 (Figure 3).

The statistical analysis showed that alarm herd status had a significant association at a 5\% significance level with the risk of being classified as a status 2 herd for all values of $c$. The $P$-values decreased from $P=$ 0.049 for $c=0$ to $P<0.0001$ for $c \geq 7$. The associated effect parameter was positive for all values of $c$, indicating that alarm herd status increased the risk of a status change.

The statistical significance at a $5 \%$ level was also evident for small jumps. Logistic regression models with jumps smaller than the threshold level $c$ as explanatory variable showed that for $3 \leq c \leq 16$, the jumps bounded by $c$ had a statistically significant effect on the probability of changing classification status from 1 to 2 . For $\mathrm{c} \geq 17$, the effect of small jumps was not statistically significant at a $5 \%$ level. For $c \leq 2$, no $P$-value could be calculated, because no jumps were to levels less than 2. Unlike for the alarm jumps, the effect parameters for these small jumps were negative for all alarm thresholds, indicating that jumps to a level below $c$ decreased the risk of a status change.

\section{DISCUSSION}

The concept of alarm herds is based on the dynamic development of antibody ODC \% measurements only, and as such it may be used as a predictor for future Salmonella Dublin herd status classification to improve early detection. This dynamic dependency, where concepts are continuously updated, is in contrast to standard applications of, for example, statistical process control (Oakland, 2008), where a (fixed) control area is set up and monitored for breaches. Furthermore, our approach differs from statistical process control in that only a part of the process that describes the ODC\% values for a herd has a stability area defined, which may change significantly over time (see Figure 1). Our interest was not directly in breaches of the stability area when it is set up, like breaching a control area in statistical process control, but rather in breaching it in 


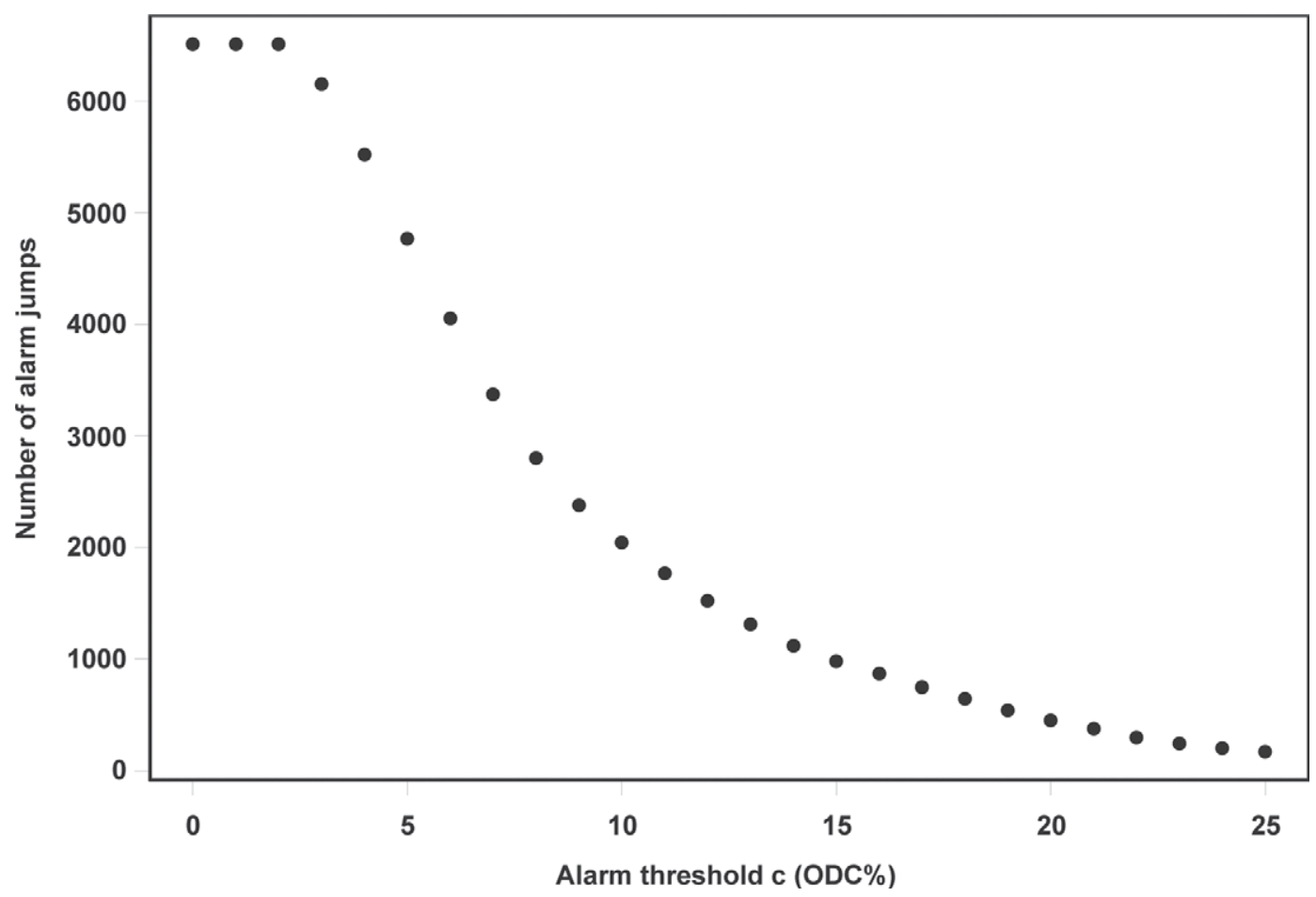

Figure 2. Number of alarm jumps that may indicate an upcoming change in Salmonella Dublin herd status from status 1 ("likely free of Salmonella Dublin") to status 2 ("likely infected with Salmonella Dublin") as a function of the alarm threshold $c$, indicating the minimum Salmonella Dublin background-corrected optical density calibrated (ODC\%) value to which the newest measurement jumped.

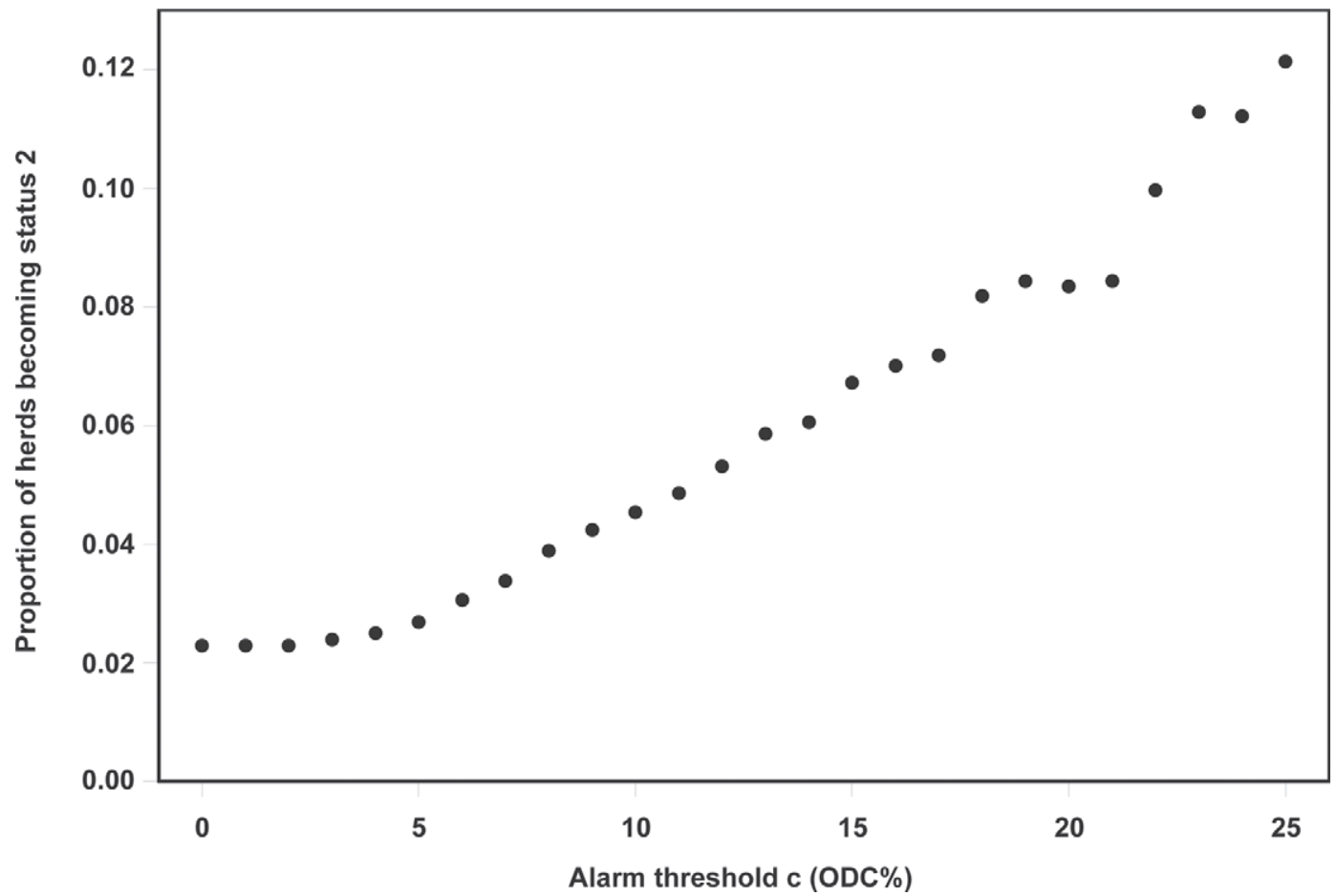

Figure 3. The proportion of alarm herds that became Salmonella Dublin status 2 ("likely infected with Salmonella Dublin") herds in the upcoming quarter as a function of the alarm threshold $c$ indicating the minimum Salmonella Dublin background-corrected optical density calibrated $(\mathrm{ODC} \%)$ value that the newest measurement jumped to. 
a specific way, by jumping above the alarm threshold. Thus, our concepts of stability areas and alarm herds are substantially different.

The predictive value is dependent on the choice of the alarm threshold, in that alarm herds for low values of $c$ had only a slightly increased risk of changing status from 1 to 2 , whereas alarm herds for $c=20$ had a 5 -fold increased risk of changing status (Figure 3), compared with the overall level of 0.016 . On the other hand, only 455 instances of an alarm jump $>20$ occurred over the study period. Hence, a trade-off exists between the number of herds being marked as alarm herds, and the level of risk that follows the concept and the associated consequences. A choice of alarm threshold should reflect this.

Although jumps bounded below a small alarm threshold appeared statistically significant, they did not increase the risk of changing status. In contrast, because the associated effect parameter was negative, it indicated that small jumps were associated with a lower probability of changing status from 1 to 2 . This may be because small jumps are associated with generally low ODC\% values. The negative effect also indicates that when considering alarm jumps as a risk factor, it is the alarm jumps to high levels, which are present for both small and high alarm thresholds, that are the major contributors to the effect on risk of changing classification status. This might be because jumps to high levels indicate true introduction and dissemination of Salmonella Dublin in the herd more frequently than do jumps to lower levels, which can more often be ascribed to variations in the bulk tank milk antibodies measurements (Nielsen and Ersbøll, 2005). Such variation may be caused, for instance, by persistently high antibody levels in the milk of some cows from a previous infection (Veling et al., 2001) or to variations in day and ELISA plate in the laboratory (Nielsen, 2002).

It is notable that for $c=17$, jumping to levels lower than this value was no longer statistically significant for predicting Salmonella Dublin status changes, indicating that herds jumping to a level less than 17 ODC\% could not be distinguished from the remaining herds. This suggests that an alarm threshold of 17 or above conforms with alarm herds being at increased risk for changing status from 1 to 2, regardless of the level at which the alarm jump ends, when alarm herd status is considered on its own and not combined with any other risk factors. This value of 17 should not be confused with the value 20 from the second criterion in the current classification system, which deals with increases from the average of the 3 preceding ODC\% values. Further research is needed to investigate the potential benefits and consequences of including the alarm herd concept in the current classification system.
The form of the alarm herd concept (i.e., the value of c) should be adjusted based on the availability of other risk factors for Salmonella Dublin status change.

It should be noted that an alarm threshold of 17 gave rise to a relative frequency of 0.072 of herds that changed status in the next quarter from 1 to 2 . Although this was more than 4 times the general risk among status 1 herds, it was far from the majority of the alarm herds that changed status in the following year-quarter. As such, alarm herds and status 2 herds are substantially different concepts. Given the economic consequences and trade restrictions that farmers may suffer due to a change to a status 2 classification, one may consider a system where an alarm herd classification triggers a remark to the farmer, notifying him/her of the risk and the need to initiate appropriate actions to prevent a possible status 2 classification 3 mo ahead. Such actions could include screening and culling of high-risk cows, improving hygienic measures, and testing of calves (Veling et. al., 2002; Nielsen and Ersbøll, 2005). It might also be of interest to investigate the risk of Salmonella Dublin classification changes associated with purchasing animals from alarm herds, because some of these are likely to be recently infected and thus could be contagious to other contact herds (Warnick et al., 2006). Further research is needed to investigate the correlation of the alarm herd concept with other risk factors for status change. An obvious route to take is to incorporate the alarm herd concept into a model that includes other known risk factors for change in herd classification, such as trade patterns and Salmonella Dublin exposure from the local environment. Another potential use of the concept may be to apply it to other diseases monitored in similar ways.

In conclusion, the alarm herd concept presented in this study provides a potential early warning method for Salmonella Dublin and other diseases or conditions that are monitored and recorded continuously over time.

\section{ACKNOWLEDGMENTS}

We are grateful to Jørgen Nielsen (Danish Cattle Federation, Århus, Denmark) for providing the necessary registry data from the Danish Cattle Database. This work was funded by the Danish Ministry of Food, Agriculture and Fisheries through the Danish Food Industry Agency (journal number: 3412-06-01634), the Milk and Cattle Levy Funds, Danish Agriculture and Meat Council (Copenhagen V, Denmark).

\section{REFERENCES}

Anonymous. 2004. Annual Report on Zoonoses in Denmark 2003. Ministry of Food, Agriculture and Fisheries, Søborg, Denmark. 
Bergevoet, R. H. M., G. van Schaik, J. Veling, G. B. C. Backus, and P. Franken. 2009. Economic and epidemiological evaluation of Salmonella control in Dutch dairy herds. Prev. Vet. Med. 89:1-7.

Carrique-Mas, J. J., J. A. Willmington, C. Papadopoulou, E. N. Watson, and R. H. Davies. 2010. Salmonella infection in cattle in Great Britain, 2003 to 2008. Vet. Rec. 167:560-565.

Ersbøll, A. K., and L. R. Nielsen. 2008. The range of influence between cattle herds is of importance for the local spread of Salmonella Dublin in Denmark. Prev. Vet. Med. 84:277-290.

Jordan, D., L. R. Nielsen, and L. D. Warnick. 2008. Modelling a national programme for the control of foodborne pathogens in livestock: The case of Salmonella Dublin in the Danish cattle industry. Epidemiol. Infect. 136:1521-1536.

Lewerin, S. S., L. Skog, J. Frössling, and H. Wahlström. 2011. Geographical distribution of salmonella infected pig, cattle and sheep herds in Sweden 1993-2010. Acta Vet. Scand. 53:51-58.

McDonough, P. L., D. Fogelman, S. J. Shin, M. A. Brunner, and D. H. Lein. 1999. Salmonella enterica serotype Dublin infection: An emerging infectious disease for the northeastern United States. J. Clin. Microbiol. 37:2418-2427.

Nielsen, L. R., and A. K. Ersbøll. 2005. Factors associated with variation in bulk-tank-milk Salmonella Dublin ELISA ODC\% in dairy herds. Prev. Vet. Med. 68:165-179.
Nielsen, L. R., L. D. Warnick, and M. Greiner. 2007. Risk factors for changing test classification in the Danish Surveillance Program for Salmonella in dairy herds. J. Dairy Sci. 90:2815-2825.

Nielsen, S. S. 2002. Variance components of an enzyme-linked immunosorbent assay for detection of IgG antibodies in milk samples to Mycobacterium avium subspecies paratuberculosis in dairy cattle. J. Vet. Med. B Infect. Dis. Vet. Public Health 49:384-387.

Oakland, J. S. 2008. Statistical Process Control. 6th ed. Elsevier, Oxford, UK.

Veling, J., H. W. Barkema, J. van der Schans, F. van Zijderveld, and J. Verhoeff. 2002. Herd-level diagnosis for Salmonella enterica ssp. enterica serovar Dublin infection in bovine dairy herds. Prev. Vet. Med. 53:31-42.

Veling, J., F. G. van Zijderveld, A. M. van Zijderveld-van Bemmel, Y. H. Schukken, and H. W. Barkema. 2001. Evaluation of two enzyme-linked immunosorbent assays for detecting Salmonella enterica ssp. enterica serovar Dublin antibodies in bulk milk. Clin. Diagn. Lab. Immunol. 8:1049-1055.

Warnick, L. D., L. R. Nielsen, J. Nielsen, and M. Greiner. 2006. Simulation model estimates of test accuracy and predictive values for the Danish Salmonella surveillance program in dairy herds. Prev. Vet. Med. 77:284-303. 